\title{
HIGH INFECTION-RELATED MORTALITY IN PAKISTANI CHILDREN WITH ACUTE LYMPHOBLASTIC LEUKAEMIA DURING REMISSION INDUCTION CHEMOTHERAPY: REVIEW OF DATA FROM A SINGLE INSTITUTION
}

\author{
Ata ur Rehman Maaz ${ }^{1}$, F. Badar'2, T. Mehmood ${ }^{3}$, I. J. Nassir ${ }^{4}$ \\ ${ }^{1}$ Department of Paediatric Oncology Children's Hospital for Wales Cardiff, United Kingdom, ${ }^{2}$ Cancer Registry, \\ Shaukat Khanum Memorial Cancer Hospital and Research Centre, Lahore, Pakistan, ${ }^{3}$ Department of Pathology, \\ Shaukat Khanum Memorial Cancer Hospital and Research Centre, Lahore, Pakistan, ${ }^{4}$ Department of Paediatric \\ Oncology, Shaukat Khanum Memorial Cancer Hospital and Research Centre, Lahore, Pakistan \\ Received: 30 March 2016 / Accepted: 20 December 2016
}

\begin{abstract}
Purpose: Despite advances in the treatment of acute lymphoblastic leukaemia (A.L.L.), the outcome for children living in the developing countries is still poor. This is in large part due to high treatment-related mortality (TRM). This study was carried out to review the data and analyze the factors resulting in high TRM during remission induction chemotherapy.

Methods: Data for children treated at our centre during the calendar year 2007 were retrospectively analysed. Standard four-drug induction chemotherapy was used without risk stratification. Bone marrow evaluation was carried out at days 8 and 28. Cerebrospinal fluid analysis was carried out on day 1 and with each subsequent intrathecal chemotherapy injection. Modern supportive care facilities including antibiotics, nutritional support and intensive care unit were available.

Results: Eighty-one children were eligible for analysis. Median age was 5 years (range 2-16), 72\% were male with M:F ratio of 2.5:1. Seventy-five (92\%) children had precursor B-cell A.L.L. Only 2 children had central nervous system leukaemia at presentation. Median presenting white blood cell count was 8.83 (range: 1-446). Severe malnutrition (weight $<5^{\text {th }}$ centile for age) was seen in $42 \%$ of children. Median symptom duration was 6 (range 1-30) weeks at the time of presentation. Induction mortality was $25 \%$. Induction mortality was $25.6 \%(\mathrm{n}=21)$. Twenty were related to infections, while more than half (52\%) occurred as a result of an outbreak of Acinetobacter infection. Severe malnutrition and Acinetobacter infection (due to an outbreak in our unit during the study period) were highly predictive of TRM during remission induction chemotherapy.
\end{abstract}

Conclusions: Infection control measures, health education and reduction in treatment intensity may improve survival for children with A.L.L. in Pakistani population.

Key words: Acute lymphoblastic leukaemia in children, malnutrition, Pakistan, treatment-related mortality

\section{Introduction}

Acute lymphoblastic leukaemia (A.L.L.) is an eminently curable cancer of children, and an increasing number of groups are reporting long-term survival in excess of $80 \%$. This success is attributable to advances in understanding of the disease biology and leukaemia cytogenetics, as

Correspondence: Dr. Ata ur Rehman Maaz, Department of Paediatric Oncology Children's Hospital for Wales Cardiff, United Kingdom.

Email: ata.maaz@wales.nhs.uk well as advances in supportive care, in addition to the refinement of treatment such as minimal residual disease (MRD) analysis..$^{[1-3]}$ The same degree of success, however, has not been duplicated in resource-poor countries. This is thought to be related to a number of factors, including delayed presentation, lack of resources for individual families and low prioritisation of cancer in some health systems. Poverty predisposes children to malnourishment which may contribute to the severity of infections when exposed to anticancer chemotherapy. The poor outcome is also attributable to delay in diagnosis, interruptions in 
treatment due to its toxicity and poor compliance with treatment. ${ }^{[4,5]}$ Lack of effective supportive care results in poor salvage rates when children experience treatmentrelated toxicity. Non-availability of sophisticated markers such as cytogenetic analysis and MRD analysis may also contribute at least to some extent to poor outcomes as these techniques can be useful in identifying children at high risk of relapse with standard treatment on the one hand and those requiring less intense therapy on the other. ${ }^{[6]}$ In most recent series in the Western literature, reported induction mortality is $<1 \%$. ${ }^{[7]}$ There are very few published studies reporting outcomes of A.L.L. from Pakistan. ${ }^{[8,9]}$ Recently published series from India have reported induction mortality between $2 \%$ and $25 \%{ }^{[10-13]}$

Shaukat Khanum Memorial Cancer Hospital and Research Centre is a philanthropically supported, modern cancer centre in Lahore, Pakistan. After an initial clinical triage process, patients are registered and treatment is provided irrespective of their ability to pay for it. A number of patients travel long distances for treatment at this centre. Approximately 350-400 new children with different paediatric cancers are registered for treatment every year at this centre. Outcome analysis for A.L.L. is beyond the scope of this study. The objective of this study was to assess the toxicity of the regimen used in the study population and analyse factors resulting in early treatment-related death.

\section{Methods}

Data were analysed retrospectively for children treated at our centre for A.L.L. during the calendar year 2007. Patients who had received prior treatment and those who presented in relapse were excluded from analysis. SPSS statistical software version 16 was used for statistical analysis. Diagnosis was confirmed with bone marrow or peripheral blood immunophenotyping of leukaemic blasts by flow cytometery. Further bone marrow examinations were undertaken on day 8 for all and day 28 for patients who had either M2, M3 or hypocellular marrows on day 8. Cerebrospinal fluid (CSF) was obtained at the time of diagnosis and disease was categorised according to the accepted groups of central nervous system (CNS) 1, 2 and 3 status. Baseline cardiac left ventricular function was estimated by multigated acquisition scan before the first dose of daunorubicin. Baseline complete blood count and biochemical profile including liver function tests, coagulation screening and serological screening for hepatitis $\mathrm{B}$ and $\mathrm{C}$ were performed at the time of diagnosis. All patients were admitted at the time of diagnosis. Induction chemotherapy was started after aggressive intravenous hydration for $24 \mathrm{~h}$ once the diagnosis of A.L.L. was established as above.

\section{Chemotherapy}

All patients received induction chemotherapy with intravenous vincristine $\left(1.5 \mathrm{mg} / \mathrm{m}^{2} /\right.$ dose on days $2,9,16$, 23 and 30), intravenous daunorubicin $\left(25 \mathrm{mg} / \mathrm{m}^{2} /\right.$ dose on days 2, 9, 16 and 23); intramuscular 1-asparaginase (Escherichia coli Asparaginase 6000 international Units/ $\mathrm{m}^{2} /$ dose on days $2,4,6,18,10,12,14,16,18,20,22$, 24 and 26) and dexamethasone $\left(6 \mathrm{mg} / \mathrm{m}^{2} /\right.$ day in two divided doses on days 1 through 28 followed by taper over 1 week). Intrathecal methotrexate was given (age-dependant dose) on days 1, 8 and 28 for CNS-negative patients and weekly for CNS-positive ones. Schedule is shown in Figure 1.

\section{Supportive care}

Institutional guidelines were followed for treating febrile neutropenia. These included broad-spectrum antibiotics, usually an antipseudomonal penicillin alongside an aminoglycoside as the first-line therapy and carbapenem as the second-line antibiotic therapy. Patient with central venous lines and those clinically suspected to have Grampositive infections also received a glycopeptides (usually teicoplanin). Antifungal cover was added for fever unresponsive by $96 \mathrm{~h}$. Antibiotic therapy was tailored for culture-proven infections according to the sensitivity of the organism. Supportive specialist advice for infectious diseases, gastroenterology and pulmonology was readily available by the on-site adult faculty. Neurology, nephrology and cardiology support was provided by visiting members of the faculty. Critically ill patients were nursed in our on-site intensive care unit where inotropic, ventilator and other interventional support was available. Product support was provided by the on-site blood bank and all blood products were screened for Hepatitis B and $\mathrm{C}$ and HIV.

\section{Results}

There were 81 evaluable patients enrolled for treatment during the study period. Median age was 5 years (range 


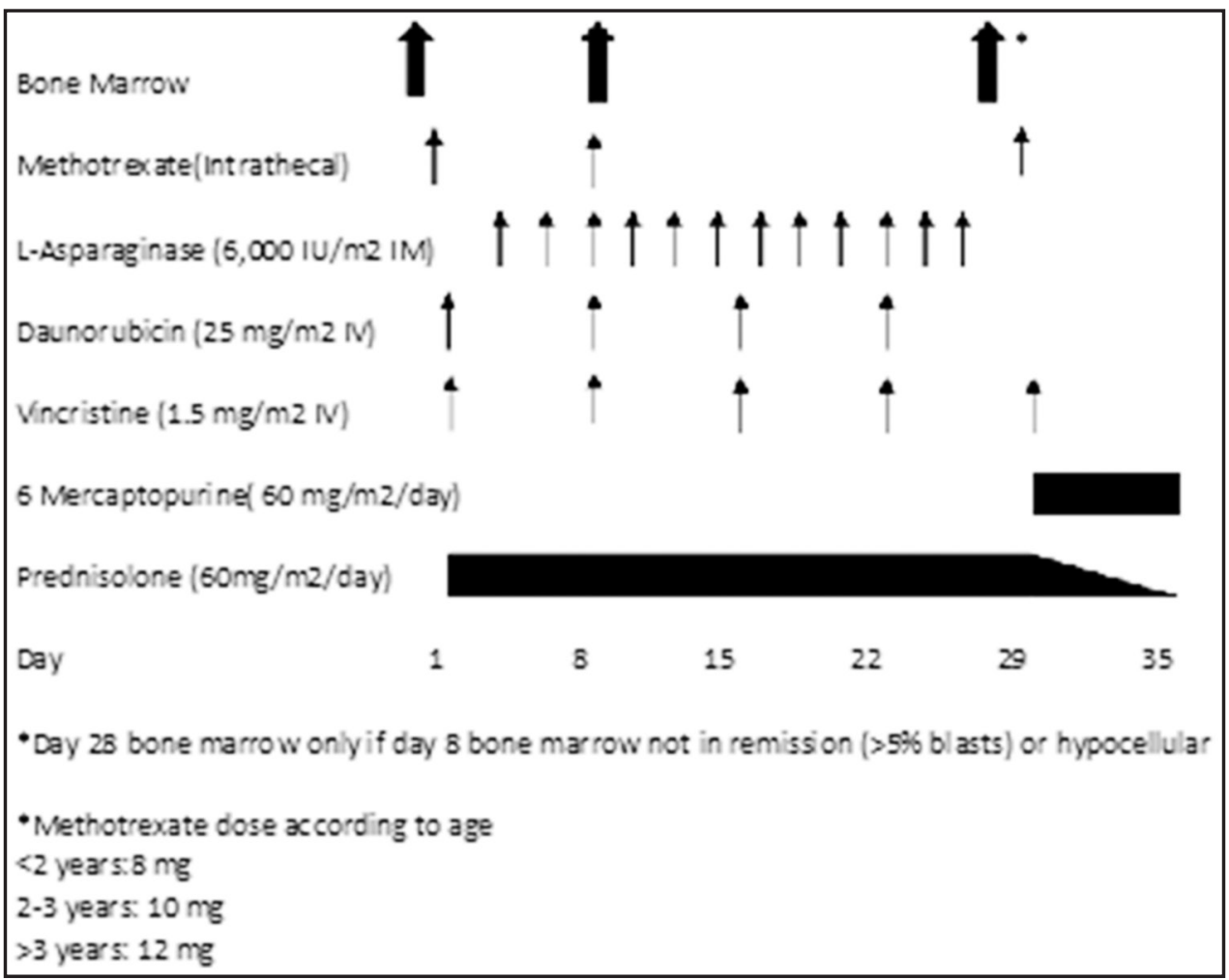

Figure 1: Treatment schema for remission induction chemotheraphy

2-16). Male-to-female ratio was 2.5:1. Seventy-five (92\%) children had precursor B cell.

A.L.L., and the remaining 6 had precursor T-cell disease. Seventy-three $(91 \%)$ children had CNS negative leukaemia, while only 2 had leukaemic blast cells in their initial CSF. Median presenting white blood cell count was 8.83 (range 1-446). Sixty-seven (82\%) children fell in NCI standard-risk group. Severe malnutrition (weight $<5^{\text {th }}$ centile for age) was seen in $42 \%$ of children. However, the median plasma albumin concentration was 4.01 (range 2.53-4.75). Median symptom duration was 6 (range 1-30) weeks at the time of presentation.

Median time to initiation of treatment (days after registration at the hospital) was 14 (3-152) days. Patient characteristics are summarised in Table 1.

\section{Response to treatment}

More than half (54\%) of children showed a rapid early response to treatment based on bone marrow morphology Table 2. Fourteen (17.3\%) had M2 and 16 (19.8\%) had M3 marrow on day 8 . By day 28 , however, all the surviving children had M1 marrow ( $<5 \%$ blasts).

\section{Toxicity}

Significant toxicities were seen in majority of children. These included Grade 3 or 4 haematological (88\%), infection-related (75\%), gastrointestinal (44\%), pulmonary (21\%), haemorrhage (13\%), liver (12\%) and neurological (7\%) toxicities.

Grade 3 and 4 haematological toxicities included prolonged neutropenia, thrombocytopenia and anaemia. Infection-related toxicities included febrile neutropenia with clinical or culture-proven infections with (Grade 4) or without (Grade 3) evidence of septic shock. These included bacterial, fungal and viral infections. Gastrointestinal toxicities included severe mucositis, esophagitis, diarrhoea, colitis and ileus. Pulmonary toxicities included X-ray-proven pneumonia, oxygen requirement and mechanical ventilation. Haemorrhagic toxicities in our patients included severe post-surgical bleeding, gastrointestinal haemorrhage and CNS bleeding. Liver 
Table 1: Patient characteristics $(n=81)$

\begin{tabular}{|c|c|c|c|c|}
\hline Characteristics & $n(\%)$ & Additional information & Median & Range \\
\hline \multicolumn{5}{|l|}{ Gender } \\
\hline Male & $58(71.6)$ & & & \\
\hline Female & $23(28.4)$ & Symptom duration (days) & 42 & $7-210$ \\
\hline Age (years) & 2-16 (Median: 5) & Time to treatment (days) & 14 & $3-152$ \\
\hline $\mathrm{NCl}$ risk group & & WBC (x109/L) & 8.83 & $1-442$ \\
\hline Standard risk & $67(82.7)$ & Albumin (mg/dL) & 4.01 & $2.53-4.75$ \\
\hline High risk & $14(17.3)$ & & & \\
\hline \multicolumn{5}{|l|}{ Lineage } \\
\hline Precursor B-cell & $75(92.6)$ & & & \\
\hline Precursor T-cell & $6(7.4)$ & & & \\
\hline \multicolumn{5}{|l|}{ Nutritional status } \\
\hline Weight $<5^{\text {th }}$ centile & $34(42)$ & & & \\
\hline Weight $>5^{\text {th }}$ centile & $47(58)$ & & & \\
\hline \multicolumn{5}{|l|}{ CSF } \\
\hline CNS1 (negative) & $73(90.1)$ & & & \\
\hline CNS2 (trauma) & $5(6.2)$ & & & \\
\hline CNS3 (positive) & $2(2.5)$ & & & \\
\hline
\end{tabular}

CSF: Cerebrospinal fluid, CNS: Central nervous system, WBC: White blood cell

Table 2: Bone marrow response at days 8 and 28

\begin{tabular}{l|c}
\hline Day 8 bone marrow* & $\boldsymbol{n}(\%)$ \\
\hline M1 & $44(54.3)$ \\
\hline M2 & $14(17.3)$ \\
\hline M3 & $16(19.8)$ \\
\hline In evaluable & $5(6.2)$ \\
\hline Day 28 bone marrow** & \\
\hline M1 & $45(100)$ \\
\hline
\end{tabular}

$M$ status refers to percentage of blasts on morphological microscopic examination of bone marrow where M1 means blast count less than 5\%, M2 5-25\% and M3 more than 25\% . *Two patients died before day 8 bone marrow examination was carried out. **Day 28 bone marrow examination was carried out for patients who were not in remission on day $8 .{ }^{* * *} \ln 15$ patients, bone marrow examination was carried out on day 28 despite having M1 marrow on day 8 to confirm continuing remission. All patients had M1 marrow on day 28

toxicities included liver failure and CNS toxicities manifested in the form of convulsions. These data are summarised in Table 3.

\section{Mortality}

There were 21 deaths in the study population during remission induction chemotherapy or soon thereafter
Table 3: Grade 3 or 4 toxicities

\begin{tabular}{l|c}
\hline Organ/system & $\boldsymbol{n}(\%)$ \\
\hline Haematological & $72(88.8)$ \\
\hline Haemorrhagic & $11(13.5)$ \\
\hline Infection & $61(75.3)$ \\
\hline GI & $36(44.4)$ \\
\hline Liver & $10(12.3)$ \\
\hline Pulmonary & $17(20.9)$ \\
\hline Neurological & $6(7.9)$ \\
\hline
\end{tabular}

(before the start of consolidation). Median time to death was 20 days (range 9-41). Twenty of the 21 deaths were infection related (Gram-negative sepsis 15; Gram-positive sepsis 2; fungal infection 2 and pneumonia 1). The remaining one death was due to an intracranial bleed. These findings are summarised in Table 4 and 5.

Nineteen of the 20 infection-related deaths had cultureproven infections. A total of 41 microorganisms were isolated (23 were Gram-negative, 12 Gram-positive bacteria while the remaining 6 were fungi). Five patients had more than one positive culture while 14 patients had a single organism isolated from their body fluids. 
Table 4: Cause of death $(n=21)$

\begin{tabular}{l|c}
\hline Cause of death & $\boldsymbol{n}(\%)$ \\
\hline Gram-negative sepsis & $15(71.4)$ \\
\hline Gram-positive sepsis & $2(9.5)$ \\
\hline Fungal infection & $2(9.5)$ \\
\hline Pneumonia & $1(4.8)$ \\
\hline Intracranial bleed & $1(4.8)$ \\
\hline
\end{tabular}

Table 5: Death analysis $(n=21 ; 25 \%)$

\begin{tabular}{|c|c|c|}
\hline Factors & $n(\%)$ & Significance \\
\hline \multicolumn{3}{|l|}{$\mathrm{NCl}$ risk group } \\
\hline $\mathrm{NCl}$ standard risk & $16(23.8)$ & $P=0.364$ \\
\hline $\mathrm{NCl}$ high risk & $5(35.7)$ & \\
\hline \multicolumn{3}{|l|}{ Morphology } \\
\hline Precursor B & $19(25.3)$ & $P=0.672$ \\
\hline Precursor T & $2(33.3)$ & \\
\hline \multicolumn{3}{|l|}{ Nutritional status } \\
\hline Not malnourished & $5(17.8)$ & $P=0.008$ \\
\hline Malnourished & $16(30.1)$ & \\
\hline \multicolumn{3}{|l|}{ Infection } \\
\hline Acinetobacter & $11(91)$ & $P=0.000$ \\
\hline No acinetobacter & $10(14)$ & \\
\hline
\end{tabular}

The most common organism isolated during the study period was Acinetobacter species (in 12 patients). All of the isolated Acinetobacter were multidrug resistant. This organism was also associated with a very high mortality (91\%). Median time from the isolation of first Acinetobacter in body fluid cultures to death was 1 day (range 0-23).

Details of these organisms are given in Table 6 and Figures 1 and 2.

Five $(35.7 \%)$ of the children in the National Cancer Institute (NCI) high-risk group died compared to $16(23.9 \%)$ in NCI standard-risk group. However, these differences were not statistically significant.

\section{Nutritional status}

Thirty-four (42\%) of children were severely malnourished i.e., weight less than $5^{\text {th }}$ centile for age according to the Tanner-Whitehouse growth charts. Median serum albumin
Table 6: Characteristics of Acinetobacter species amongst the dead $(n=12)$

\begin{tabular}{l|c}
\hline Characteristic & $\boldsymbol{n}(\%)$ \\
\hline Body fluid & $8(75)$ \\
\hline Blood & $4(25)$ \\
\hline Tracheal aspirate & \\
\hline Sensitivity pattern & $12(100)$ \\
\hline Multidrug resistant & 0 \\
\hline Sensitive & \\
\hline Blood counts & $7(58)$ \\
\hline Neutrophils $<1.0$ & $5(42)$ \\
\hline Neutrophils $>1.0$ & \\
\hline Location & $7(58)$ \\
\hline ICU & $4(33)$ \\
\hline Ward & $1(9)$ \\
\hline Outpatient & \\
\hline
\end{tabular}

ICU: Intensive care unit

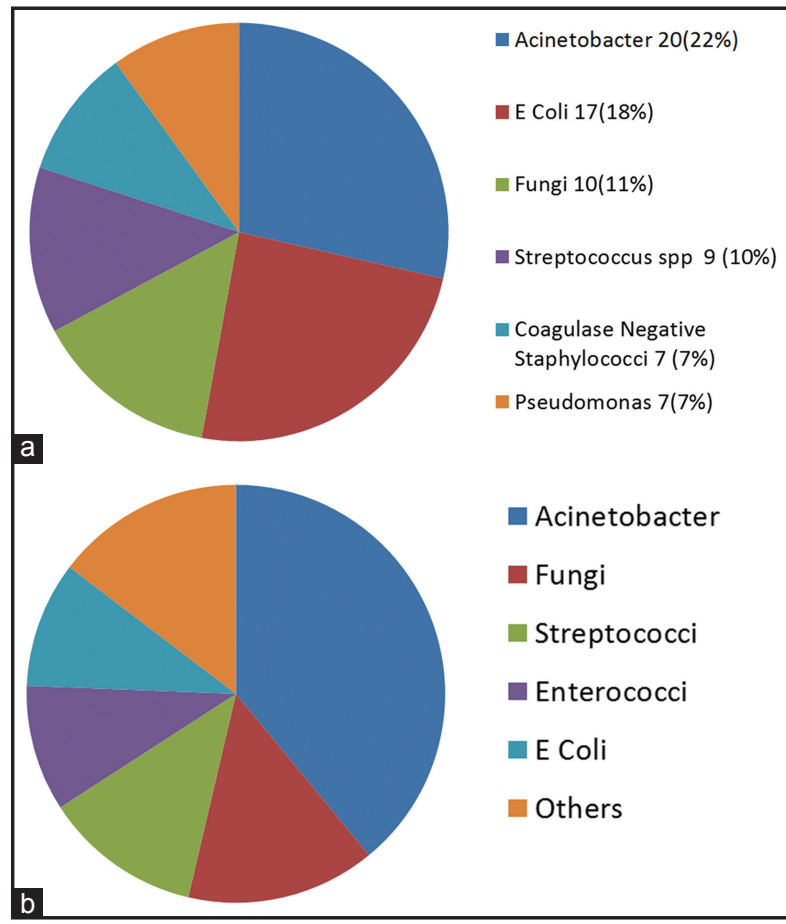

Figure 2: (a) Microorganisms isolated during induction $(n=90)$. (b) Microorganisms isolated from patients who later died $(n=41)$

concentration was $4.01 \mathrm{~g} / \mathrm{dL} .16(47 \%)$ of severely malnourished children died compared to 5 (8.6\%) who were not severely malnourished. Severe malnourishment was significantly correlated to the risk of induction 
mortality $(P=0.008)$. However, a lower serum albumin was not correlated to mortality in our series.

\section{Discussion}

There are limited data on disease characteristics and outcomes of A.L.L. for Pakistani children. ${ }^{[8,9]}$ To our knowledge, this is the first study from Pakistan reporting treatmentrelated mortality (TRM) for ALL induction therapy. Aziz et al. analysed the prognostic implication of presentation characteristics and outcome analysis using a modified BFM protocol in 2002. Reported induction mortality was in keeping with other published data from this part of the world. Their sample size was small, making it difficult to draw any definitive conclusions. ${ }^{[8]}$ Khalid et al. have recently described a single-centre experience over 18 years using six different treatment protocols. Their starting sample size was 96 . However, $52 \%$ were excluded for various reasons, leaving a small sample. Some of the traditional prognostic factors were confirmed while others were not found to be of any significance. They do not describe TRM. ${ }^{[9]}$

In the absence of consensus local guidelines and prospective cooperative trials, treatment of A.L.L. in Pakistan and indeed in many other developing countries of the world has historically been modelled on the templates of recognised international protocols. A standard four-drug chemotherapy regimen was used for all children during the remission induction phase without risk stratification. Template of the intermediate-risk arm of the UKALL 2003 study was followed, which was the current (at the time) CCLG UK protocol.

The results of our study show that there was a much higher than expected mortality (25.9\%) in this population. This was primarily related to an outbreak of Acinetobacter infection during the study period. This organism, while of low virulence in the immunocompetent, is associated with a high mortality in the immunocompromised and seriously ill patients. ${ }^{[14]}$ Our study population, which was at increased risk of mortality due to severe malnourishment and exposure to myelosuppressive chemotherapy, contracting Acinetobacter was highly predictive of mortality.

Successful implementation of infection control measures has since brought the incidence and resulting in high mortality under control (hospital data, personal communication). If all the Acinetobacter cases are excluded from the analysis, induction mortality in the remaining group would be $11 \%$ that is more in keeping with other studies from the region.

Poor nutritional reserves, poor personal hygiene and overcrowded living conditions all contribute to poor chemotherapy tolerance, severe immune suppression and early death. Displacement from hometown has a dual impact on family incomes (i.e. losing primary income and incurring additional expense by staying away from home). Our results show significantly higher induction mortality in severely malnourished group. Others have also reported high TRM in malnourished children and in children belonging to poor socioeconomic and educational backgrounds. ${ }^{[5,15-17]}$

For several years, the poor outcome of childhood A.L.L. in this part of the world has been assumed to be at least partly related to adverse cytogenetic changes, hence the tendency of treatment with intensive regimens without stratification. Siraj et al. reported a higher incidence of adverse cytogenetics and a lower incidence of favourable translocation in Indian children with A.L.L. compared to Europeans and Americans. Faiz and Qazi also reported a less than expected favourable translocation $t$ (12; 21) in Pakistani population. ${ }^{[18,19]}$ However, even the highest estimates place the adverse cytogenetics group around $7-8 \%$, which would still mean that over $90 \%$ of children could possibly be stratified to receive a lower intensity treatment. The importance of risk stratification is thus highlighted based on conventional, molecular and cytogenetic criteria to avoid over or under treatment.

Glucocorticoids are an essential part of A.L.L. treatment. Through the past many decades, prednisolone has been used by all major cooperative groups. Much is known about its efficacy and toxicity. Over the recent years, studies have shown that dexamethasone to be superior due to a relatively increased CNS penetration and reduction in the risk of CNS and bone marrow relapse. Mitchell et al. demonstrated that risk of CNS and non-CNS relapse in the group randomised to dexamethasone was half that of the prednisolone group in a randomised fashion. However, it is also known that dexamethasone is associated with increased risk of infections and other organ-system 
toxicities. ${ }^{[20]}$ Increased mortality individually as well as in combination with daunorubicin has been reported. ${ }^{[21,22]}$ Inclusion of dexamethasone in the four-drug regimen may have had a compound contributory effect to the high toxicity profile of this regimen in our population.

We conclude that strict infection control measures are required to reduce induction mortality for A.L.L. Health education regarding improving hygiene and reducing overcrowding is paramount to reduce the infections and related mortality. Toxicity of treatment and induction mortality can be brought down by low-intensity regimen to low- and standard-risk patients. These changes have already been introduced and will need to be examined in future studies.

\section{Conflict of Interest}

The authors declare that they have no conflict of interest.

\section{References}

1. Pui CH, Robison LL, Look AT. Acute lymphoblastic leukaemia. Lancet 2008;371:1030-43.

2. Pui CH, Evans WE. Treatment of acute lymphoblastic leukemia. N Engl J Med 2006;354:166-78.

3. Silverman LB, Gelber RD, Dalton VK, et al. Improved outcome for children with acute lymphoblastic leukemia: Results of Dana-Farber consortium protocol 91-01. Blood 2001; 97:1211-8.

4. Mejía-Aranguré JM, Fajardo-Gutiérrez A, Reyes-Ruíz NI, et al. Malnutrition in childhood lymphoblastic leukemia: A predictor of early mortality during the induction-toremission phase of the treatment. Arch Med Res 1999; 30:150-3.

5. Gupta S, Bonilla M, Fuentes SL, et al. Incidence and predictors of treatment-related mortality in paediatric acute leukaemia in el salvador. Br J Cancer 2009;100:1026-31.

6. de Haas V, Breunis WB, Verhagen OJ, et al. Accurate quantification of minimal residual disease at day 15 , by real-time quantitative polymerase chain reavtion identifies also patients with B-precursor acute lymphoblastic leukemia at high risk for relapse. Blood 2000;96:1619-20.

7. Pui CH, Sandlund JT, Pei D, et al. Improved outcome for children with acute lymphoblastic leukemia: Results of total therapy study XIIIB at St Jude children's research hospital. Blood 2004; 104:2690-6.

8. Aziz Z, Zahid M, Mahmood R, et al. Modified BFM protocol for childhood acute lymphoblastic leukemia: A retrospective analysis. Med Pediatr Oncol 1997;28:48-53.

9. Khalid S, Moiz B, Adil SN, et al. Retrospective review of pediatric patients with acute lymphoblastic leukemia: A single center experience. Indian J Pathol Microbiol 2010; 53:704-10.

10. Gao YJ, Lu FJ, Wang HS. Treating childhood acute lymphoblastic leukemia in a developing country 1998-2003: The experience of a single children's hospital in China. J Pediatr Hematol Oncol 2006;28:798-802.

11. Advani S, Pai S, Venzon D, et al. Acute lymphoblastic leukemia in India: An analysis of prognostic factors using a single treatment regimen. Ann Oncol 1999;10:167-76.

12. Magrath I, Shanta V, Advani S, et al. Treatment of acute lymphoblastic leukaemia in countries with limited resources; lessons from use of a single protocol in India over a twenty year period [corrected]. Eur J Cancer 2005;41:1570-83.

13. Kulkarni KP, Marwaha RK, Trehan A, et al. Survival outcome in childhood ALL: Experience from a tertiary care centre in north India. Pediatr Blood Cancer 2009;53:168-73.

14. Gales AC, Jones RN, Forward KR, et al. Emerging importance of multidrug-resistant acinetobacter species and Stenotrophomonas maltophilia as pathogens in seriously ill patients: Geographic patterns, epidemiological features, and trends in the SENTRY antimicrobial surveillance program (1997-1999). Clin Infect Dis 2001;32 Suppl 2:S104-13.

15. Barr RD, Gibson BE. Nutritional status and cancer in childhood. J Pediatr Hematol Oncol 2000;22:491-4.

16. Barr RD, Ruiz-Argüelles GJ. Nutrition and cancer in children. Med Pediatr Oncol 2003;41:54-7.

17. Pinkel D. Selecting treatment for children with acute lymphoblastic leukemia. J Clin Oncol 1996;14:4-6.

18. SirajAK, KamatS, GutiérrezMI, etal. Frequencies of the major subgroups of precursor B-cell acute lymphoblastic leukemia in Indian children differ from the west. Leukemia 2003; 17:1192-3.

19. Faiz M, Qazi JI. T(12:21) is underrepresented in childhood B-lineage acute lymphoblastic leukemia in Punjab, Pakistan. J Pediatr Hematol Oncol 2010;32:249-51.

20. Mitchell CD, Richards SM, Kinsey SE, et al. Benefit of dexamethasone compared with prednisolone for childhood acute lymphoblastic leukaemia: Results of the UK medical research councilALL97 randomized trial. Br J Haematol 2005; 129:734-45.

21. Belgaumi AF, A1-Bakrah M, Al-Mahr M, et al. Dexamethasone-associated toxicity during induction chemotherapy for childhood acute lymphoblastic leukemia is augmented by concurrent use of daunomycin. Cancer 2003; 97:2898-903.

22. Hurwitz CA, Silverman LB, Schorin MA, et al. Substituting dexamethasone for prednisone complicates remission induction in children with acute lymphoblastic leukemia. Cancer 2000;88:1964-9. 